\title{
An Analysis of the Replicator Dynamics for an Asymmetric Hawk-Dove Game
}

\author{
Ikjyot Singh Kohli and Michael C. Haslam \\ Department of Mathematics and Statistics, York University, Toronto, ON, Canada \\ Correspondence should be addressed to Ikjyot Singh Kohli; isk@mathstat.yorku.ca
}

Received 5 January 2017; Accepted 28 March 2017; Published 24 April 2017

Academic Editor: Patricia J. Y. Wong

Copyright ( 2017 Ikjyot Singh Kohli and Michael C. Haslam. This is an open access article distributed under the Creative Commons Attribution License, which permits unrestricted use, distribution, and reproduction in any medium, provided the original work is properly cited.

\begin{abstract}
We analyze, using a dynamical systems approach, the replicator dynamics for the asymmetric Hawk-Dove game in which there is a set of four pure strategies with arbitrary payoffs. We give a full account of the equilibrium points and their stability and derive the Nash equilibria. We also give a detailed account of the local bifurcations that the system exhibits based on choices of the typical Hawk-Dove parameters $v$ and $c$. We also give details on the connections between the results found in this work and those of the standard two-strategy Hawk-Dove game. We conclude the paper with some examples of numerical simulations that further illustrate some global behaviours of the system.
\end{abstract}

\section{Introduction}

The Hawk-Dove game is one of the first examples of a pairwise game that was used to model the conflict between animals [1]. The basic idea is that "Hawks" and "Doves" represent two types of behaviours (actions or pure strategies) that could be exhibited by animals of the same species [2]. In the standard Hawk-Dove game, individuals can use one of two possible pure strategies. In one case, they can be aggressive/a "Hawk," which is typically denoted by $\mathrm{H}$, or be nonaggressive/a "Dove," which is typically denoted by D. Then, at various times, individuals in this population can have a conflict over a resource which has value $v$, where the winner of the conflict gets the resource and the loser pays a cost $c$.

The Hawk-Dove game has been studied in the context of replicator dynamics a number of times over the past several years. Some examples of these studies include [3-17].

In replicator dynamics, it is assumed that individuals are programmed to use only pure strategies from a finite set $\mathbf{S}=$ $\left\{s_{1}, \ldots, s_{k}\right\}$. It can be shown [2] that the dynamical evolution of the proportion of individuals using strategy $s_{i}, x_{i}$, is given by

$$
\dot{x}_{i}=\left[\pi\left(s_{i}, \mathbf{x}\right)-\bar{\pi}(\mathbf{x})\right] x_{i},
$$

where $\pi\left(s_{i}, \mathbf{x}\right)$ is the payoff to individuals using strategy $s_{i}$, while $\bar{\pi}(\mathbf{x})$ is known as the average payoff and is defined as

$$
\bar{\pi}(\mathbf{x})=\sum_{i=1}^{k} x_{i} \pi\left(s_{i}, \mathbf{x}\right) .
$$

Further to (1), one also has the constraint

$$
\sum_{i=1}^{k} x_{i}=1 .
$$

In this paper, we wish to consider an asymmetric pairwise Dove-Hawk game. Following [2], specifically, this is where two individuals are contesting ownership of a territory that one of them controls. One assumes that the value of the territory and costs of contest are the same for both players. Unlike the standard Hawk-Dove game described above, players can now condition their behaviour on the role that they occupy, which is typically denoted as owner or intruder. So, the pure strategies now take the forms play Hawk if owner and play Dove if intruder, which we will be denoted by HD. Therefore, there is a set of four pure strategies:

$$
\mathbf{S}=\{\mathrm{HH}, \mathrm{HD}, \mathrm{DH}, \mathrm{DD}\} .
$$


TABLE 1: The payoff matrix for the asymmetric Hawk-Dove game.

\begin{tabular}{lccc}
\hline & $\mathrm{HH}$ & $\mathrm{HD}$ & $\mathrm{DH}$ \\
\hline $\mathrm{HH}$ & $\frac{(v-c)}{2}, \frac{(v-c)}{2}$ & $\frac{(3 v-c)}{4}, \frac{(v-c)}{4}$ & $\frac{(3 v-c)}{4}, \frac{(v-c)}{4}$ \\
$\mathrm{HD}$ & $\frac{(v-c)}{4}, \frac{(3 v-c)}{4}$ & $\frac{v}{2}, \frac{v}{2}$ & $\frac{(2 v-c)}{4}, \frac{(2 v-c)}{4}$ \\
$\mathrm{DH}$ & $\frac{(v-c)}{4}, \frac{(3 v-c)}{4}$ & $\frac{(2 v-c)}{4}, \frac{(2 v-c)}{4}$ & $\frac{v}{2}, \frac{v}{2}$ \\
$\mathrm{DD}$ & $0, v$ & $\frac{v}{4}, \frac{3 v}{4}$ & $\frac{v}{4}, \frac{3 v}{4}$ \\
\hline
\end{tabular}

From these arguments, it can be shown [2] that the payoff matrix is given by Table 1 .

We note that the replicator dynamics of this four-strategy asymmetric Hawk-Dove game have not been analyzed from a dynamical systems perspective in the literature to the best of the authors' knowledge. However, some examples of related asymmetric games can be found in [18-26].

\section{The Dynamical Equations}

Let us denote by $(x, y, z, w)$ the proportion of individuals who use strategies $\mathrm{HH}, \mathrm{HD}, \mathrm{DH}$, and DD, respectively. Then, from the payoff matrix in Table 1 and (1)-(2), the replicator dynamics are given by the following dynamical system:

$$
\begin{aligned}
\dot{x} & =\frac{1}{4} x[-c(2 x+y+z)-4 \bar{\pi}(\mathbf{x}) \\
& +v(4 w+2 x+3(y+z))], \\
\dot{y} & =\frac{1}{4} y[-c(x+z)-4 \bar{\pi}(\mathbf{x})+v(3 w+x+2(y+z))], \\
\dot{z} & =\frac{1}{4} z[-c(x+y)-4 \bar{\pi}(\mathbf{x})+v(3 w+x+2(y+z))], \\
\dot{w} & =\frac{1}{4} w[v(2 w+y+z)-4 \bar{\pi}(\mathbf{x})],
\end{aligned}
$$

where

$$
\bar{\pi}(\mathbf{x})=\frac{1}{2}[v-c(x+y)(x+z)],
$$

and, from (3),

$$
x+y+z+w=1
$$

This four-dimensional dynamical system can be reduced to three dimensions if we set, via (7), $w=1-x-y-z$.
Therefore, in what follows, we will study the following unconstrained three-dimensional system:

$$
\begin{aligned}
\dot{x} & =\frac{1}{4} x\left[c\left(2 x^{2}+2 x(y+z-1)+2 y z-y-z\right)\right. \\
& -v(2 x+y+z-2)], \\
\dot{y}= & -\frac{1}{4} y[v(2 x+y+z-1) \\
& -c(2 x+2 y-1)(x+z)], \\
\dot{z} & =-\frac{1}{4} z[v(2 x+y+z-1)-c(x+y)(2 x+2 z-1)] .
\end{aligned}
$$

\section{A Local Stability Analysis}

From (8), we now present the equilibrium points along with their eigenvalues and local stability. The Jacobian matrix, denoted by $J_{i j}$, corresponding to this dynamical system is a $3 \times 3$ matrix, whose entries are listed as follows:

$$
\begin{aligned}
J_{11} & =\frac{1}{4}\left(c\left(6 x^{2}+4 x(y+z-1)+2 y z-y-z\right)\right. \\
& -v(4 x+y+z-2)) \\
J_{12} & =\frac{1}{4} x(c(2 x+2 z-1)-v) \\
J_{13} & =\frac{1}{4} x(c(2 x+2 y-1)-v) \\
J_{21} & =\frac{1}{4} y(c(4 x+2 y+2 z-1)-2 v) \\
J_{22} & =\frac{1}{4}(c(2 x+4 y-1)(x+z) \\
& -v(2 x+2 y+z-1))
\end{aligned}
$$

$J_{23}=\frac{1}{4} y(c(2 x+2 y-1)-v)$,

$J_{31}=\frac{1}{4} z(c(4 x+2 y+2 z-1)-2 v)$,

$J_{32}=\frac{1}{4} z(c(2 x+2 z-1)-v)$, 


$$
\begin{aligned}
J_{33} & =\frac{1}{4}(c(x+y)(2 x+4 z-1) \\
& -v(2 x+y+2 z-1)) .
\end{aligned}
$$

3.1. Equilibrium Point 1. The first equilibrium point was found to be

$$
P_{1}:(x, y, z)=(0,0,1) .
$$

The corresponding eigenvalues of $J_{i j}$ were found to be

$$
\left\{\lambda_{1}, \lambda_{2}, \lambda_{3}\right\}=\left\{-\frac{v}{4},-\frac{c}{4}, \frac{v-c}{4}\right\}
$$

This point is a stable node if

$$
\{v>0\} \cap\{c>v\} .
$$

It is an unstable node if

$$
\{v<0\} \cap\{c<v\} .
$$

It is a saddle point if

$$
\begin{aligned}
& \{\{v<0\} \cap\{v<c<0\}\} \cup\{\{v<0\} \cap\{c>0\}\} \\
& \quad \cup\{\{v>0\} \cap\{c<0\}\} \cup\{\{v>0\} \cap\{0<c<v\}\} .
\end{aligned}
$$

3.2. Equilibrium Point 2. The second equilibrium point was found to be

$$
P_{2}:(x, y, z)=\left(0, \frac{1}{2}, \frac{1}{2}\right)
$$

The corresponding eigenvalues of $J_{i j}$ were found to be

$$
\left\{\lambda_{1}, \lambda_{2}, \lambda_{3}\right\}=\left\{\frac{c}{8}, \frac{1}{8}(c-2 v), \frac{1}{8}(2 v-c)\right\} .
$$

This point is neither a stable nor an unstable node. However, it is a saddle point under the following conditions:

$$
\begin{aligned}
& \{(v \leq 0 \cap c<2 v) \cup(v>0 \cap c<0)\} \\
& \quad \cup\{v>0 \cap 0<c<2 v\} \cup\{v<0 \cap 2 v<c<0\} .
\end{aligned}
$$

3.3. Equilibrium Point 3. The third equilibrium point was found to be

$$
P_{3}:(x, y, z)=\left(0, \frac{v}{c}, \frac{v}{c}\right) .
$$

The corresponding eigenvalues of $J_{i j}$ were found to be

$$
\left\{\lambda_{1}, \lambda_{2}, \lambda_{3}\right\}=\left\{\frac{v}{4},-\frac{v(c-2 v)}{4 c}, 0\right\}
$$

The single zero eigenvalue indicates that this equilibrium point is normally hyperbolic, and the local stability can be determined through the nonzero eigenvalues by the invariant manifold theorem [27]. In particular, this point is a stable node if

$$
v<0 \cap 2 v<c<0 .
$$

It is an unstable node if

$$
v>0 \cap 0<c<2 v .
$$

It is a saddle point under the following conditions:

$$
\begin{aligned}
& \{v<0 \cap(c<2 v \cup c>0)\} \\
& \quad \cup\{v>0 \cap(c<0 \cup c>2 v)\} .
\end{aligned}
$$

3.4. Equilibrium Point 4. The fourth equilibrium point was found to be

$$
P_{4}:(x, y, z)=(0,1,0) \text {. }
$$

The corresponding eigenvalues of $J_{i j}$ were found to be

$$
\left\{\lambda_{1}, \lambda_{2}, \lambda_{3}\right\}=\left\{-\frac{v}{4},-\frac{c}{4}, \frac{v-c}{4}\right\}
$$

This point is a stable node if

$$
\{v>0\} \cap\{c>v\} .
$$

It is an unstable node if

$$
\{v<0\} \cap\{c<v\} .
$$

It is a saddle point under the following conditions:

$$
\begin{aligned}
& \{v<0 \cap v<c<0\} \cup\{v<0 \cap c>0\} \\
& \quad \cup\{v>0 \cap c<0\} \cup\{v>0 \cap 0<c<v\} .
\end{aligned}
$$

3.5. Equilibrium Point 5. The fifth equilibrium point was found to be

$$
P_{5}:(x, y, z)=(1,0,0) \text {. }
$$

The corresponding eigenvalues of $J_{i j}$ were found to be

$$
\left\{\lambda_{1}, \lambda_{2}, \lambda_{3}\right\}=\left\{\frac{c-v}{2}, \frac{c-v}{4}, \frac{c-v}{4}\right\}
$$

This point is a stable node if

$$
\{v \in \mathbb{R}\} \cap\{c<v\} .
$$

It is an unstable node if

$$
\{v \in \mathbb{R}\} \cap\{c>v\} .
$$

From (29), it can be seen that $\mathscr{P}_{5}$ is in fact never a saddle point of the dynamical system. 
3.6. Equilibrium Point 6. The sixth equilibrium point was found to be

$$
P_{6}:(x, y, z)=\left(\frac{v}{c}, 0,0\right) .
$$

The corresponding eigenvalues of $J_{i j}$ were found to be

$$
\left\{\lambda_{1}, \lambda_{2}, \lambda_{3}\right\}=\left\{0,0, \frac{v(v-c)}{2 c}\right\} .
$$

One sees that since $\lambda_{1}=\lambda_{2}=0$, this point is manifestly nonhyperbolic. As such, its stability properties cannot be determined through the Jacobian matrix.

3.7. Equilibrium Point 7. The final equilibrium point was found to be

$$
P_{7}:(x, y, z)=(0,0,0) .
$$

The corresponding eigenvalues of $J_{i j}$ were found to be

$$
\left\{\lambda_{1}, \lambda_{2}, \lambda_{3}\right\}=\left\{\frac{v}{2}, \frac{v}{4}, \frac{v}{4}\right\} .
$$

This point is a stable node if

$$
v<0 .
$$

It is an unstable node if

$$
v>0 .
$$

Further, this point is never a saddle point as can be seen from (35).

\section{Local Bifurcations}

With knowledge of the equilibrium points and their local stability as given in the previous sections, we now attempt to describe bifurcation behaviour exhibited by this dynamical system. Analyzing bifurcation behaviour is important as this determines the local changes in stability of the equilibrium points of the system.

The mechanism for these bifurcations can be seen as follows. form

The linearized system in a neighbourhood of $P_{1}$ takes the

$$
\begin{aligned}
& \dot{x}=\frac{1}{4} x(v-c), \\
& \dot{y}=-\frac{1}{4} y c, \\
& \dot{z}=\frac{1}{4} x(c-2 v)+\frac{c-v}{4} y-\frac{v}{4} z .
\end{aligned}
$$

We see that $x$ destabilizes $P_{1}$ when $v=c, y$ destabilizes $P_{1}$ when $c=0$, and $z$ destabilizes $P_{1}$ when $v=c=0$.
The linearized system in a neighbourhood of $P_{2}$ takes the form

$$
\begin{aligned}
& \dot{x}=\frac{1}{8} x(2 v-c), \\
& \dot{y}=\frac{1}{8} x(c-2 v)+\frac{1}{8} y(c-v)-\frac{1}{8} v z, \\
& \dot{z}=\frac{1}{8} x(c-2 v)-\frac{1}{8} y v+\frac{1}{8}(c-v) z .
\end{aligned}
$$

We see that $x$ destabilizes $P_{2}$ along the line $c=2 v$, while $y$ and $z$ destabilize $P_{2}$ when $v=c=0$.

The linearized system in a neighbourhood of $P_{3}$ takes the form

$$
\begin{aligned}
& \dot{x}=0, \\
& \dot{y}=-\frac{v(c-2 v)}{4 c} x+\frac{v^{2}}{4 c} y+\frac{v(v-c)}{4 c} z, \\
& \dot{z}=-\frac{v(c-2 v)}{4 c} x+\frac{v(v-c)}{4 c} y+\frac{v^{2}}{4 c} z .
\end{aligned}
$$

Therefore, $P_{3}$ is destabilized by $y$ and $z \forall c \neq 0, v=0$.

The linearized system in a neighbourhood of $P_{4}$ takes the form

$$
\begin{aligned}
& \dot{x}=\frac{1}{4} x(v-c), \\
& \dot{y}=\frac{1}{4}(c-2 v) x-\frac{v}{4} y+\frac{c-v}{4} z, \\
& \dot{z}=-\frac{c}{4} z .
\end{aligned}
$$

Therefore, $x$ destabilizes $P_{4}$ along the line $v=c$. Further, $y$ destabilizes $P_{4}$ when $v=c=0$. Finally, $z$ destabilizes $P_{4}$ when $c=0$ for $v \in \mathbb{R}$.

The linearized system in a neighbourhood of $P_{5}$ takes the form

$$
\begin{aligned}
& \dot{x}=\frac{c-v}{2} x+\frac{c-v}{4} y+\frac{c-v}{4} z, \\
& \dot{y}=\frac{c-v}{4} y, \\
& \dot{z}=\frac{c-v}{4} z .
\end{aligned}
$$

Therefore, $P_{5}$ is destabilized by $x, y$, and $z$ along the line $c=v$. form

The linearized system in a neighbourhood of $P_{6}$ takes the

$$
\begin{aligned}
& \dot{x}=\frac{v(v-c)}{2 c} x+\frac{v(v-c)}{4 c} y+\frac{v(v-c)}{4 c}, \\
& \dot{y}=0, \\
& \dot{z}=0 .
\end{aligned}
$$

Therefore, $x$ destabilizes $P_{6}$ whenever $v=0$, or whenever $v=$ $c($ for $c \neq 0)$. 

form

The linearized system in a neighbourhood of $P_{7}$ takes the

$$
\begin{aligned}
& \dot{x}=\frac{v}{2} x, \\
& \dot{y}=\frac{v}{4} y, \\
& \dot{z}=\frac{v}{4} z .
\end{aligned}
$$

We see that, therefore, $P_{7}$ is destabilized by $x, y$, and $z$ whenever $v=0$, for $c \in \mathbb{R}$. From these calculations, we can therefore see that, along $v=c$, as one goes from $c<0$ to $c>0, P_{1}$ and $P_{4}$ go from being unstable nodes to stable ones, and vice versa, while $P_{3}$ goes from being a stable node to an unstable one. Whenever $c=0, v \in \mathbb{R}$, as one goes from $v<0$ to $v>0, P_{5}$ goes from being an unstable node to a stable node, while $P_{7}$ goes from being a stable node to an unstable one. Along the line $c=2 v$, as we go from $v<0$ to $v>0, P_{1}$ and $P_{4}$ go from being unstable nodes to stable nodes, while $P_{5}$ and $P_{7}$ go from being stable nodes to unstable nodes. Finally, whenever $v=0, c \in \mathbb{R}$, as we go from $c<0$ to $c>0, P_{5}$ goes from being a stable node to an unstable one, while $P_{1}$ and $P_{4}$ go from being unstable nodes to stable ones.

\section{Nash Equilibria}

Determining the future asymptotic behaviour of the replicator dynamics is of importance since, by Theorem 9.15 in [2], if $\mathbf{x}^{*}$ is an asymptotically stable fixed point of the dynamical system, then the symmetric strategy pair $\left[\sigma^{*}, \sigma^{*}\right]=\left[\mathbf{x}^{*}, \mathbf{x}^{*}\right]$ is a Nash equilibrium.

Following [28], we note that, first, by Lyapunov's theorem, if all eigenvalues of the linear part of a vector field $v$ at a singular point have a negative real part, the singular point is asymptotically stable.

From our stability analysis of the various equilibrium points in the preceding sections, we therefore observe the following Nash equilibria of the replicator dynamics depending on the choices of $v$ and $c$ :

(1) $v>0, c>v \Rightarrow P_{1}$ is asymptotically stable $\Rightarrow$ $[(0,0,1),(0,0,1)]$ is a Nash equilibrium.

(2) $v>0, c>v \Rightarrow P_{4}$ is asymptotically stable $\Rightarrow$ $[(0,1,0),(0,1,0)]$ is a Nash equilibrium.

(3) $v \in \mathbb{R}, c<v \Rightarrow P_{5}$ is asymptotically stable $\Rightarrow$ $[(1,0,0),(1,0,0)]$ is a Nash equilibrium.

(4) $c \in \mathbb{R}, v<0 \Rightarrow P_{7}$ is asymptotically stable $\Rightarrow$ $[(0,0,0),(0,0,0)]$ is a Nash equilibrium.

The existence of these Nash equilibria shows that this asymmetric Hawk-Dove game produces rational behaviour in a population composed of players that are not required to make consciously rational decisions. In other words, the population is stable when, given what everyone else is doing, no individual would get a better result by adopting a different strategy. This is the so-called population view of a Nash equilibrium, which Nash himself described as the mass action interpretation $[2,29]$.
TABle 2: Payoff matrix for the standard two-strategy Hawk-Dove game.

\begin{tabular}{lcc}
\hline & $\mathrm{H}$ & $\mathrm{D}$ \\
\hline $\mathrm{H}$ & $\frac{(v-c)}{2}, \frac{(v-c)}{2}$ & $v, 0$ \\
$\mathrm{D}$ & $0, v$ & $\frac{v}{2}, \frac{v}{2}$ \\
\hline
\end{tabular}

\section{Connections with the Two-Strategy Hawk-Dove Game}

It is perhaps of interest to discuss our results found above in connection with the standard two-strategy Hawk-Dove game. Following [2], we note that the payoff matrix for such a game is given by Table 2 .

In this case, the replicator dynamics are a simple consequence of (1)-(2). Namely, let $z$ denote the proportion of individuals in the population that use strategy $\mathrm{H}$ in Table 2. Then, the replicator dynamics are governed by the single ordinary differential equation

$$
\dot{z}=\frac{c}{2} z(1-z)\left(\frac{v}{c}-z\right) .
$$

Clearly, (45) has equilibrium points $z=0, z=1$, and $z=v / c$. Let us denote by $f(z)$ the right-hand side of (45). Then,

$$
f^{\prime}(z)=\frac{1}{2}[v-2 v z+c z(-2+3 z)] .
$$

Clearly, when $z=0, f^{\prime}(z)=v / 2$, which is negative when $v<0$ and positive when $v>0$. Therefore, the point $z=0$ is a stable node when $v<0$ and an unstable node when $v>0$. Further, when $z=1, f^{\prime}(z)=(c-v) / 2$. In this case, the point $z=0$ is a stable node for $c \in \mathbb{R}$ and $v>c$. Further, it is an unstable node for $c \in \mathbb{R}$ and $v<c$. Finally, when $z=v / c$, we have that $f^{\prime}(z)=v(v-c) / 2 c$. This point is a stable node when $v<0$ and $v<c<0$, or when $v>0$ and $c<0$ or $c>v$. It is an unstable node when $v<0$ and $c<v$ or $c>0$, or when $v>0$ and $0<c<v$.

Comparing these cases to the Nash equilibria we found in the full asymmetric game, we see that the case when $z=0$ corresponds to the case of Equilibrium Point 7, where $[(0,0,0),(0,0,0)]$ was a Nash equilibrium. The case $z=v / c$ in this example corresponds to Equilibrium Points 1 and 4 , where $[(0,0,1),(0,0,1)]$ and $[(0,1,0),(0,1,0)]$ were both found to be Nash equilibria of the full asymmetric replicator dynamics. Certainly, this shows that $z \rightarrow v / c$ for any initial population that is not at an equilibrium point.

\section{Some Numerical Simulations}

In this section, we present some numerical simulations of the work above. These simulations were completed in MATLAB using the ODE23s solver with a variety of initial conditions which are denoted with asterisks in Figures 1, 2, 3, 4, and 5.

In Figure 1, we assume that $v=0.1, c=0.2$; in Figure 2, we assume that $v=0.2, c=0.3$; in Figure 3, we assume that $v=0.2, c=0.1$; in Figure 4, we assume that $v=-0.1, c=0.2$; and in Figure 5, $v=-0.2, c=-0.1$. 


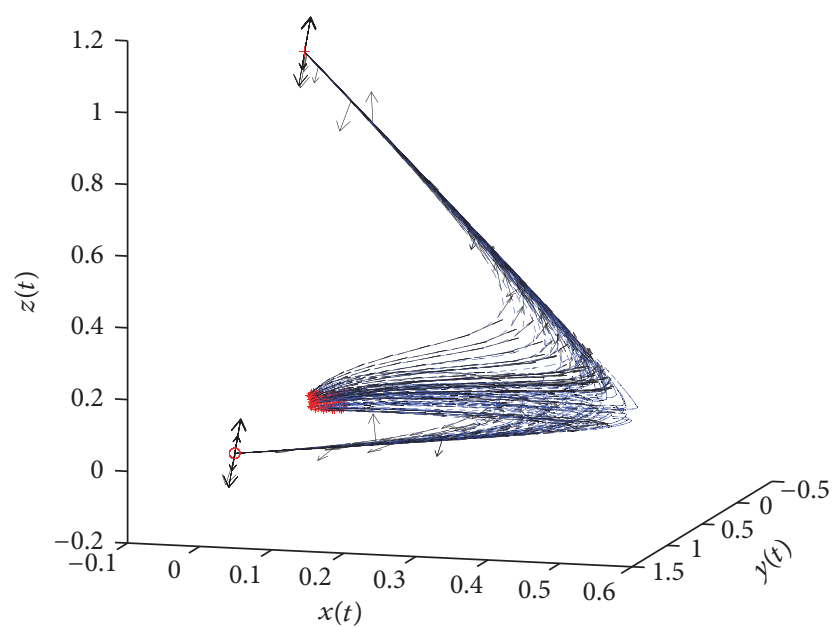

FIGURE 1: Results where $v=0.1, c=0.2$. The red cross denotes the equilibrium point $P_{1}:(x, y, z)=(0,0,1)$, and the red circle denotes the equilibrium point $P_{4}:(x, y, z)=(0,1,0)$.

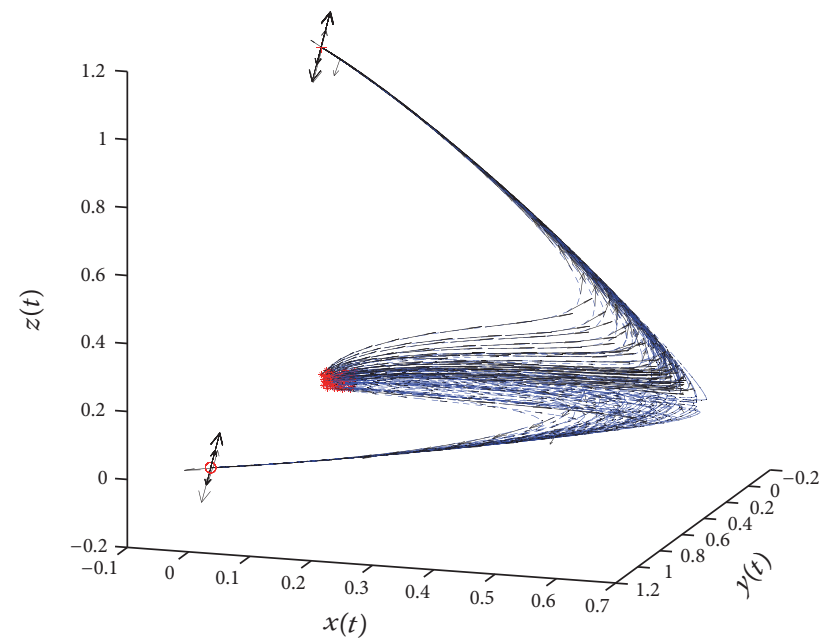

FIgURE 2: Results where $v=0.2, c=0.3$. The red cross denotes the equilibrium point $P_{1}:(x, y, z)=(0,0,1)$, and the red circle denotes the equilibrium point $P_{4}:(x, y, z)=(0,1,0)$.

\section{Conclusions}

In this paper, we analyzed, using a dynamical systems approach, the replicator dynamics for the asymmetric HawkDove game in which there is a set of four pure strategies with arbitrary payoffs. We gave a full account of the equilibrium points and their stability and derived the Nash equilibria. In particular, we found that if $v>0, c>0$, then the strategy pairs [HD, HD] and [DH, DH] are Nash equilibria. If $v \in \mathbb{R}$, $c<v$, then the strategy pair $[\mathrm{HH}, \mathrm{HH}]$ is a Nash equilibrium. Finally, if $c \in \mathbb{R}, v<0$, then the strategy pair [DD, DD] is a Nash equilibrium. We also gave a detailed account of the local bifurcations that the system exhibits based on choices of the typical Hawk-Dove parameters $v$ and $c$. We also gave details on the connections between the results we found and those of the standard two-strategy Hawk-Dove game. We concluded

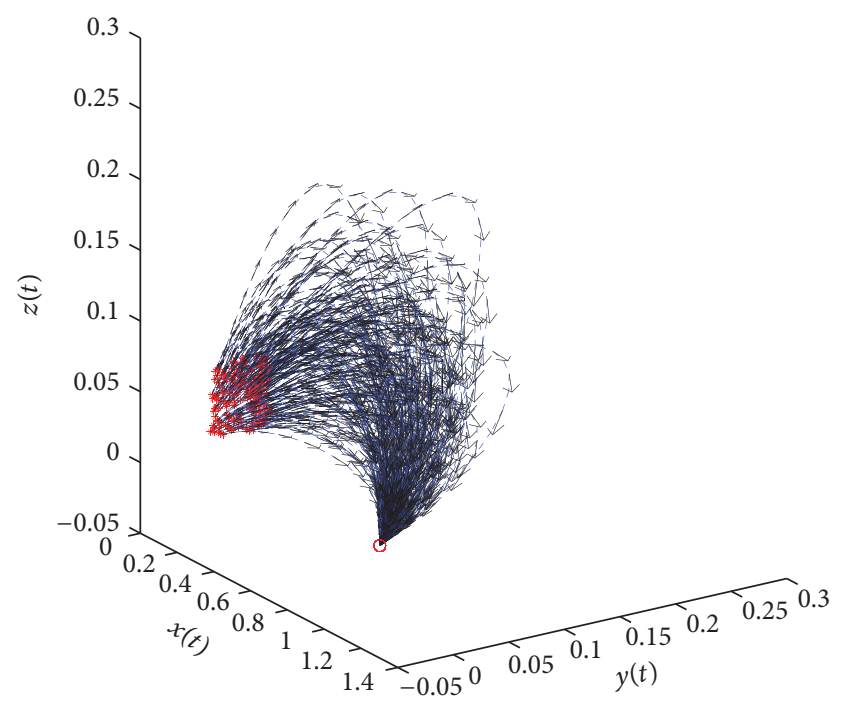

FIGURE 3: Results where $v=0.2, c=0.1$. The red circle denotes the equilibrium point $P_{5}:(x, y, z)=(1,0,0)$.

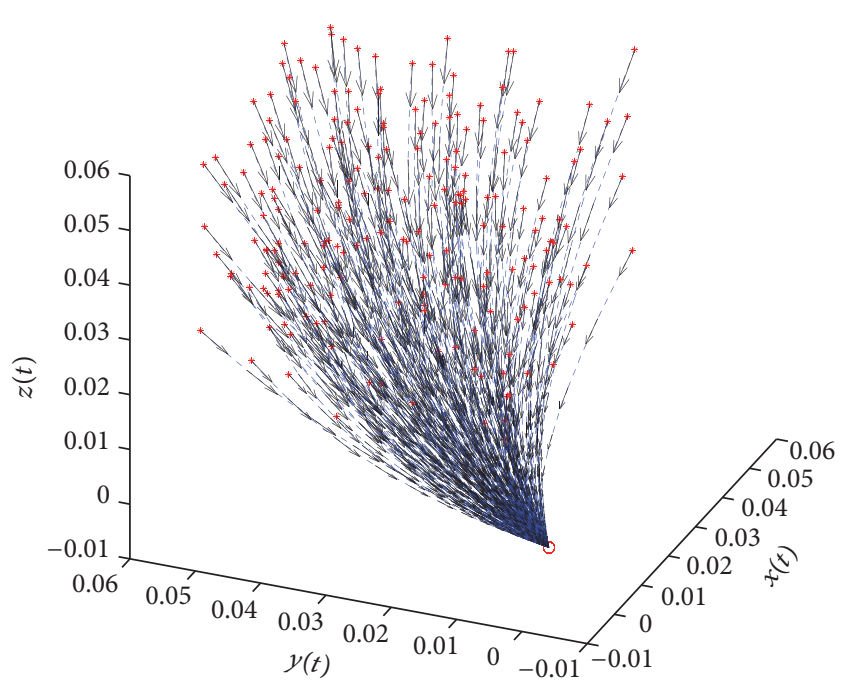

FIGURE 4: Results where $v=-0.1, c=0.2$. The red circle denotes the equilibrium point $P_{7}:(x, y, z)=(0,0,0)$.

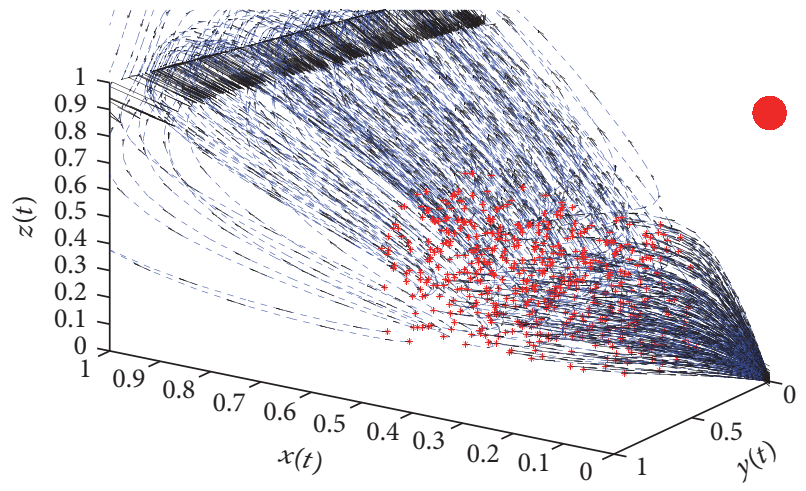

FIGURE 5: Results where $v=-0.2, c=-0.1$. The shaded red circle denotes the equilibrium point $P_{1}:(x, y, z)=(0,0,1)$. One can see that, indeed, under these choices for $v$ and $c, P_{1}$ is indeed a saddle point as predicted by our stability analysis. 
the paper with some examples of numerical simulations that further illustrate some global behaviours of the system.

\section{Conflicts of Interest}

The authors declare that they have no conflicts of interest.

\section{Acknowledgments}

This research was partially supported by a grant given to Michael C. Haslam from the Natural Sciences and Engineering Research Council of Canada.

\section{References}

[1] J. M. Smith, Evolution and the Theory of Games, Cambridge University Press, 1982.

[2] J. N. Webb, Game Theory: Decisions, Interaction and Evolution, Springer, 2007.

[3] I. M. Bomze, "Lotka-Volterra equation and replicator dynamics: a two-dimensional classification," Biological Cybernetics, vol. 48, no. 3, pp. 201-211, 1983.

[4] C. P. Roca, J. A. Cuesta, and A. Sánchez, "Evolutionary game theory: temporal and spatial effects beyond replicator dynamics," Physics of Life Reviews, vol. 6, no. 4, pp. 208-249, 2009.

[5] C. Taylor, D. Fudenberg, A. Sasaki, and M. A. Nowak, "Evolutionary game dynamics in finite populations," Bulletin of Mathematical Biology, vol. 66, no. 6, pp. 1621-1644, 2004.

[6] S. G. Ficici and J. B. Pollack, "A game-theoretic approach to the simple coevolutionary algorithm," in Proceedings of the International Conference on Parallel Problem Solving from Nature, pp. 467-476, Springer, 2000.

[7] L. Samuelson, "Evolution and game theory," Journal of Economic Perspectives, vol. 16, no. 2, pp. 47-66, 2002.

[8] D. Friedman, "On economic applications of evolutionary game theory," Journal of Evolutionary Economics, vol. 8, no. 1, pp. 1543, 1998.

[9] D. Fudenberg, M. A. Nowak, C. Taylor, and L. A. Imhof, "Evolutionary game dynamics in finite populations with strong selection and weak mutation," Theoretical Population Biology, vol. 70, no. 3, pp. 352-363, 2006.

[10] R. A. Johnstone, "Eavesdropping and animal conflict," Proceedings of the National Academy of Sciences of the United States of America, vol. 98, no. 16, pp. 9177-9180, 2001.

[11] S. R. X. Dall, A. I. Houston, and J. M. McNamara, "The behavioural ecology of personality: consistent individual differences from an adaptive perspective," Ecology Letters, vol. 7, no. 8, pp. 734-739, 2004.

[12] J. Hofbauer and K. Sigmund, Evolutionary Games and Population Dynamics, Cambridge University Press, 1998.

[13] B. Wydick, Games in Economic Development, Cambridge University Press, 2007.

[14] E. Ahmed and A. S. Hegazi, "On dynamical multi-team and signaling games," Applied Mathematics and Computation, vol. 172, no. 1, pp. 524-530, 2006.

[15] M. Doebeli and C. Hauert, "Models of cooperation based on the Prisoner's Dilemma and the Snowdrift game," Ecology Letters, vol. 8, no. 7, pp. 748-766, 2005.

[16] A. Traulsen, J. M. Pacheco, and M. A. Nowak, "Pairwise comparison and selection temperature in evolutionary game dynamics," Journal of Theoretical Biology, vol. 246, no. 3, pp. 522-529, 2007.

[17] J. W. Weibull, Evolutionary Game Theory, MIT Press, 1997.

[18] M. Mesterton-Gibbons, "Ecotypic variation in the asymmetric Hawk-Dove game: when is Bourgeois an evolutionarily stable strategy?" Evolutionary Ecology, vol. 6, no. 3, pp. 198-222, 1992.

[19] S. Matsumura and T. Kobayashi, "A game model for dominance relations among group-living animals," Behavioral Ecology and Sociobiology, vol. 42, no. 2, pp. 77-84, 1998.

[20] M. Nakamaru and A. Sasaki, "Can transitive inference evolve in animals playing the hawk-dove game?" Journal of Theoretical Biology, vol. 222, no. 4, pp. 461-470, 2003.

[21] J.-Z. He, R.-W. Wang, and Y.-T. Li, "Evolutionary stability in the asymmetric volunteer's dilemma," PLoS ONE, vol. 9, no. 8, Article ID e103931, 2014.

[22] A. McAvoy and C. Hauert, "Asymmetric evolutionary games," PLoS Computational Biology, vol. 11, no. 8, Article ID e1004349, 2015.

[23] T. Uehara and Y. Iwasa, "Global mutations and local mutations have very different effects on evolution, illustrated by mixed strategies of asymmetric binary games," Journal of Theoretical Biology, vol. 262, no. 2, pp. 223-231, 2010.

[24] T. Sekiguchi and H. Ohtsuki, "Fixation probabilities of strategies for bimatrix games in finite populations," Dynamic Games and Applications, vol. 7, no. 1, pp. 93-111, 2017.

[25] A. Benz, G. Jäger, and R. Van Rooij, Game Theory and Pragmatics, Springer, 2005.

[26] R. Cressman, Evolutionary Dynamics and Extensive Form Games, vol. 5, MIT Press, 2003.

[27] J. Wainwright and G. Ellis, Dynamical Systems in Cosmology, Cambridge University Press, 1st edition, 1997.

[28] D. Anosov, S. K. Aranson, V. Arnold, I. Bronshtein, V. Grines, and Y. Il'yashenko, Ordinary Differential Equations and Smooth Dynamical Systems, vol. 3rd, Springer, 1997.

[29] J. Nash, Non-cooperative games [Ph.D. dissertation], Princeton University, 1950. 


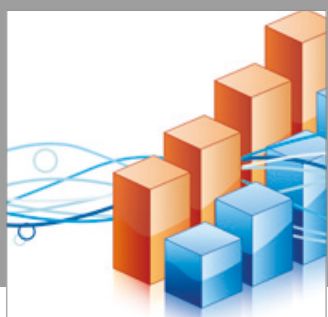

Advances in

Operations Research

vatersals

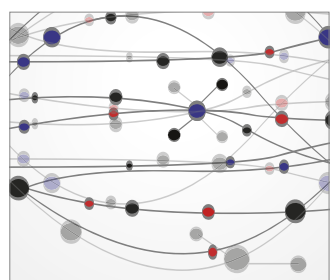

\section{The Scientific} World Journal
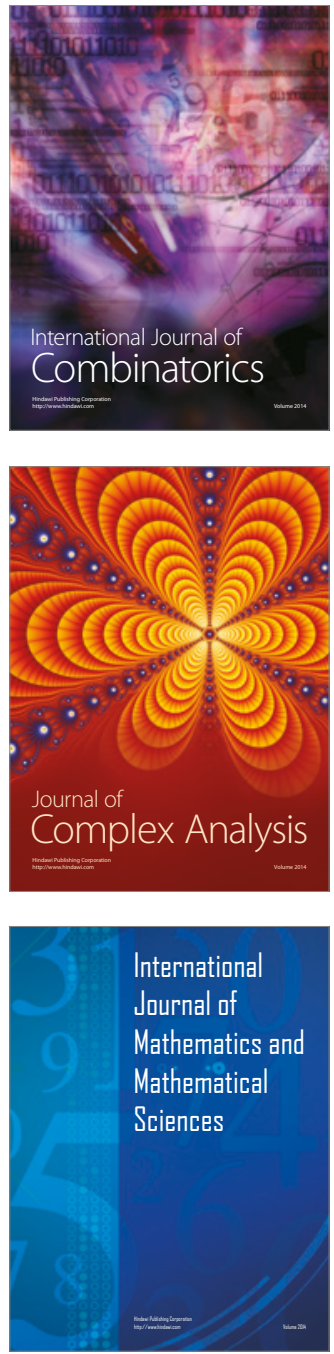
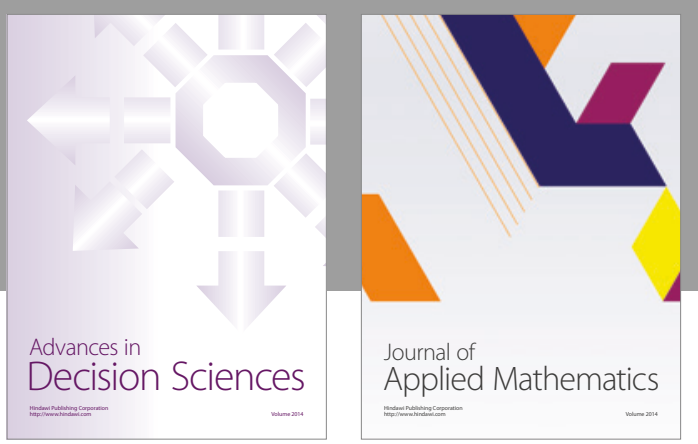

Algebra

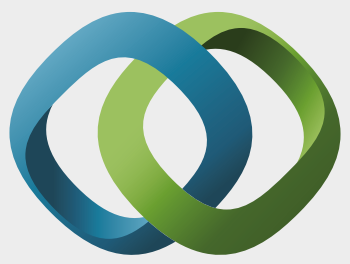

\section{Hindawi}

Submit your manuscripts at

https://www.hindawi.com
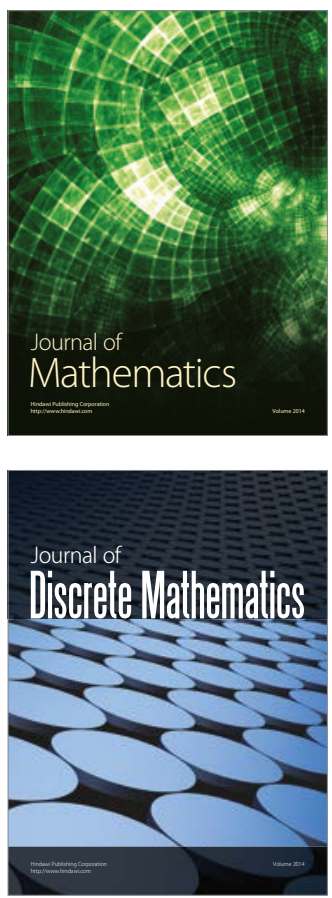

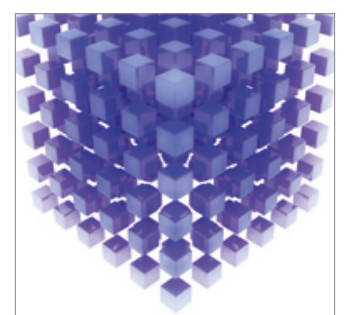

Mathematical Problems in Engineering
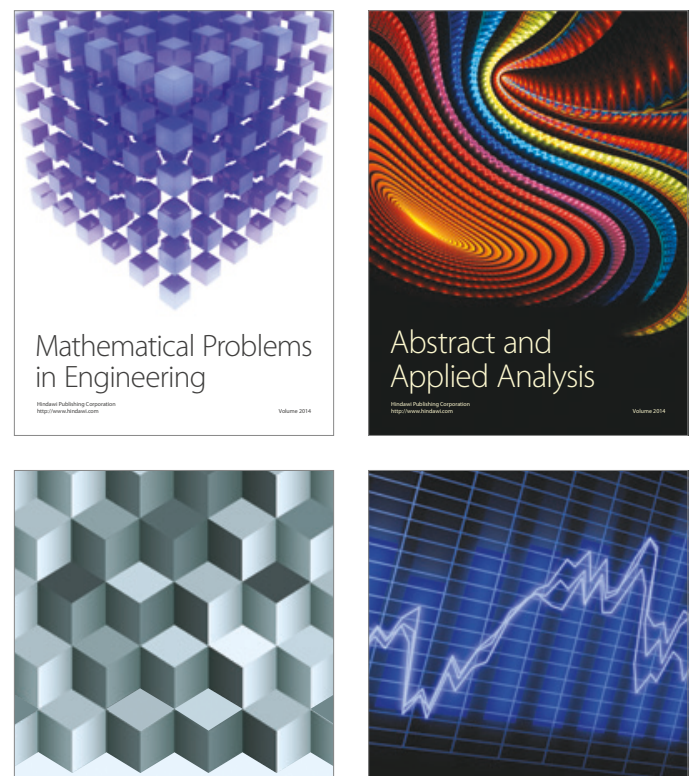

Journal of

Function Spaces

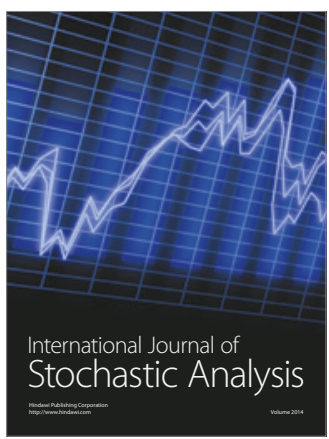

Probability and Statistics
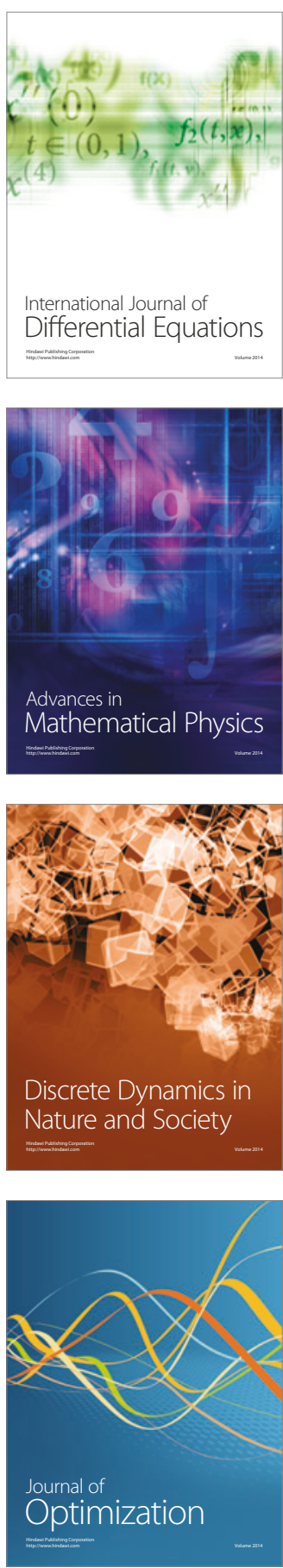